\title{
Characterization of Carbohydrate Structures of Bovine MUC15 and Distribution of the Mucin in Bovine Milk
}

\author{
L. T. Pallesen, L. R. L. Pedersen, T. E. Petersen, and J. T. Rasmussen ${ }^{1}$ \\ Protein Chemistry Laboratory, Department of Molecular Biology, University of Aarhus, 8000 Aarhus C, Denmark
}

\begin{abstract}
The present work reports the characterization of carbohydrate structures and the distribution of the newly identified mucin MUC15, a highly glycosylated protein associated with the bovine milk fat globule membrane (MFGM). Distribution of MUC15 was investigated in various fractions of bovine milk by densitometric scanning of Western blots. In raw milk, MUC15 was shown to constitute $0.08 \%$ (wt) of the protein and approximately $1.5 \%$ (wt) of the MFGM-associated proteins. Surprisingly, this study showed that in addition to the fat-containing fractions, such as MFGM and buttermilk, MUC15 was present in nonfat-containing fractions as well, such as skim milk and whey. Compositional and structural studies of the carbohydrates of bovine milk MUC15 showed that the glycans are composed of fucose, galactose, mannose, $\mathrm{N}$-acetylgalactosamine, $N$-acetylglycosamine, and sialic acid. The carbohydrate was shown to constitute $65 \%$ of the total molecular weight, and the molar ratios of the individual sugars to protein of the O-linked glycans were determined. The glycan structures of MUC15 were further studied by enzymatic deglycosylation experiments using different endo- and exoglycosidases as well as a panel of lectins. The N-linked glycans were shown to contain mainly hybrid-type $\mathrm{N}$-glycans. In addition, the $\mathrm{N}$-glycans were shown to be sialylated and contain terminal poly-lactosamine structures. The O-linked glycans were found to constitute some unsubstituted Core1 structures and a substantial number of sialylated Core-1 O-linked glycans. By comparing the results of peanut agglutinin lectin binding, enzymatic deglycosylation, and monosaccharide composition analysis, we concluded that bovine MUC15 also contains more complex O-glycans containing high amounts $\mathrm{N}$-acetylglucosamine residues. Furthermore, a small subset of the O-linked glycans is decorated with lactosamine on their terminal ends.
\end{abstract}

Received February 6, 2007.

Accepted March 6, 2007.

${ }^{1}$ Corresponding author: jatr@mb.au.dk
Key words: mucin, milk fat globule membrane, glycosylation, distribution

\section{INTRODUCTION}

The mucins constitute a heterogeneous family of large, filamentous, and heavily glycosylated proteins present at the apical surface of many epithelial cells. Many important biological processes, including protection of epithelial surfaces, immune responses, adhesion, inflammation, and tumor genesis, appear to be afforded or modulated by mucins or mucin-like glycoproteins (for a review see Moniaux et al., 2001). MUC15 is one of the 20 human mucins that have been identified thus far (for references see Rose and Voynow, 2006). Originally, MUC15 was described as a high molecular weight glycoprotein (PAS III) associated with the milk fat globule membrane (MFGM) of bovine milk (Mather et al., 1980). Isolation and characterization of the $130-\mathrm{kDa}$ glycoprotein from bovine MFGM revealed that it was a previously unknown protein and was classified as a mucin-type glycoprotein (Pallesen et al., 2002). Mucins are divided into at least 2 distinct subfamilies: the secreted (gel-forming and non-gel-forming) mucins and the membrane-bound mucins. The 307 AA-residue-long mature MUC15 belongs to the latter group, with one membrane-spanning domain, a short intracellular Cterminal region of 74 residues, and a large extracellular N-terminal region rich in Ser, Thr, and Pro residues (41\%; Pallesen et al., 2002).

Mucins are synthesized as rod-shaped apomucin cores that are posttranslationally modified by exceptionally abundant O-glycosylation of Ser and Thr residues by glycans linked through $N$-acetylgalactosamine (GalNAc). Bovine MUC15 contains large amounts of covalently linked carbohydrate, as evidenced by its ability to be stained with periodic acid Schiff's (PAS) stain and by the large divergence between the calculated molecular mass of mature MUC15, at 33,317 $\mathrm{Da}$, and the approximately $130 \mathrm{kDa}$ extrapolated from the electrophoretic mobility. Interestingly, 11 of 15 potential Nglycosylation motifs have been shown to be glycosylated on MUC15 isolated from bovine milk (Pallesen et al., 2002). Although the carbohydrates of mucins are 
mainly O-linked, N-glycosylations have been demonstrated in other mucins as well, and the surface localization of MUC17 has been suggested to depend on Nglycosylation of the mucin (Ho et al., 2003).

In contrast to $\mathrm{N}$-glycosylation, no consensus recognition sequence for the O-glycosyltransferases has been formulated. The influence of peptide sequence and environment on the mucin-type O-glycosylation pattern is not fully understood. Gerken et al. (2002) found that neighboring glycosylation status can be a significant factor modulating the first step of O-glycan biosynthesis. Furthermore, many studies have noted a skew in AA composition around mucin-type O-glycosylation sites (Christlet and Veluraja, 2001), with a higher frequency of Pro, Ser, and Thr residues than expected, as observed for MUC15 and other mucins.

The present work describes the distribution and quantification of MUC15 in bovine milk as well as in various fractions thereof and samples from the dairy industry. Moreover, the study presents a characterization of the carbohydrate moieties, including quantitative compositional analyses and structural studies of both N- and O-linked glycans of bovine milk MUC15, by enzymatic deglycosylation using endo- and exoglycosidases and lectin-blotting experiments.

\section{MATERIALS AND METHODS}

\section{Materials}

Polyclonal antibodies against bovine milk MUC15 were recovered from rabbit antibovine MUC15 serum (manufactured by DakoCytomation, Glostrup, Denmark) by use of a protein A Sepharose column (GE Healthcare, Little Chalfont, UK) and were subsequently affinity-purified on a Hi-Trap NHS-activated HP column (GE Healthcare) coupled with bovine MUC15. Precision Plus Protein Dual Color Standards for SDS-PAGE were from Bio-Rad Laboratories (Hercules, CA). The Digoxigenin (DIG) Glycan Differentiation kit was purchased from Roche Diagnostics and the Enzymatic Protein Deglycosylation kit (E-DEGLY kit) was from Sigma (St. Louis, MO). All other chemicals were of analytical grade.

\section{Milk Fractions}

All fractions were made from a pool of fresh, unpasteurized milk (raw milk). Skim milk, buttermilk, the supernatant of acidified buttermilk, and MFGM were prepared essentially as described by Hvarregaard et al. (1996). Whey was obtained by lowering the $\mathrm{pH}$ of skim milk to 4.6 and subsequently removing precipitated $\mathrm{CN}$ by centrifugation. In addition, samples were obtained by ultracentrifugation $(141,000 \times g, 3 \mathrm{~h})$ of skim milk (serum, skim milk membranes, and CN; Heegaard et al., 1997) and buttermilk (supernatant of ultracentrifuged buttermilk and MFGM by ultracentrifugation) as previously reported (Benfeldt et al., 1995). Protein concentrations of all collected bovine milk samples were determined by a modification of the method of Lowry (Schacterle and Pollack, 1973). Fractions from the dairy industry were also examined, including buttermilk from the butter-making machine, whey from yellow cheese production, and dry milk powders, that is, whey protein concentrate (Lacprodan DI 8090), whey protein isolate (Lacprodan DI 9212), and whey fat concentrate (synonymous with macromolecular whey protein or Lacprodan MFGM 10). Whey fat concentrate equals the retentate obtained by microfiltration $(0.1$ to $0.2 \mu \mathrm{m})$ of whey from yellow cheese production (which equals whey protein concentrate when spray-dried), and whey protein isolate is the permeate from this process. Dry milk powders were solubilized in water prior to analysis. The dairy milk fractions and dry milk powders were produced and kindly donated by Arla Foods Ingredients, Nr. (Virum, Denmark).

\section{Quantification of MUC15 in Milk Fractions}

Quantities of different milk samples and bovine MUC15 standards were separated by SDS-PAGE (18\% polyacrylamide gels) and electrotransferred to Hybond$\mathrm{P}$ polyvinylidene difluoride (PVDF) membranes (GE Healthcare) in a wet transfer for $1 \mathrm{~h}$ [10 $\mathrm{mM}$ 3-(cyclohexylamino)-1-propanesulfonic acid, 10\% methanol, and $0.005 \%$ SDS] with a 500-mA constant current. Western blotting was performed as described by Benfeldt et al. (1995) using primary polyclonal antibodies against bovine MUC15 diluted to $0.5 \mu \mathrm{g} / \mathrm{mL}$ and alkaline phosphatase-conjugated swine-antirabbit secondary antibodies (DakoCytomation) diluted 1:3,000. The density of individual bands was quantified by aid of a flat-bed scanner and QuantiScan 2.1 software (Biosoft, Cambridge, UK). Linearity between the amount of bovine milk MUC15 and staining density was obtained in a range from 8 to $31 \mathrm{ng} /$ lane. The bovine MUC15 used as a standard was purified from bovine milk as described previously (Pallesen et al., 2002) and was quantified by AA analysis (o-phthaldialdehyde-based).

\section{O-Sialoglycoprotein Endopeptidase Susceptibility}

Native bovine MUC15 was dissolved in $50 \mathrm{mM}$ HEPES, $\mathrm{pH} 7.4$, to $3 \mu \mathrm{g} / \mu \mathrm{L}$ and incubated at $37^{\circ} \mathrm{C}$ with $0.11 \mu \mathrm{g} / \mu \mathrm{L}$ of $\mathrm{O}$-sialoglycoprotein endopeptidase from Mannheimia haemolytica (Cedarlane Laboratories Ltd., Hornby, Canada). Samples were taken at $15 \mathrm{~min}$, $2.5 \mathrm{~h}$, and $18 \mathrm{~h}$, electrophoresed on 18\% SDS-polyacryl- 
amide gels, and transferred to Hybond-P PVDF membranes. Western blotting was performed as described using polyclonal antibodies against bovine MUC15.

\section{Enzymatic Deglycosylation}

MUC15 was enzymatically deglycosylated using the E-DEGLY kit (Sigma). The kit contains 5 different endo- and exoglycosidases needed to completely remove all N-linked and simple O-linked carbohydrates from glycoproteins, as well as more complex Core-2 O-linked carbohydrates. The enzymes include peptide:N-glycosidase F (PNGase F); Chryseobacterium (Flavobacterium) meningosepticum], O-glycosidase (recombinant from Streptococcus pneumoniae), $\alpha$-2(3,6,8,9)-neuraminidase (recombinant from Arthrobacter ureafaciens), $\beta(1-4)$-galactosidase (recombinant from Strep. pneumoniae), and $\beta-N$-acetylglucosaminidase (recombinant from Strep. pneumoniae). Deglycosylation was carried out essentially as described by the manufacturer. Briefly, bovine MUC15 was dissolved in distilled water, mixed with $250 \mathrm{mM}$ sodium phosphate, $\mathrm{pH} 6.0$ (3:1 vol/ vol), and denatured with SDS at $100^{\circ} \mathrm{C}$ for $5 \mathrm{~min}$. When cooled to room temperature, Triton X-100 (15\% solution) was added ( $16: 1 \mathrm{vol} / \mathrm{vol})$. The enzymes were added and the samples were subsequently incubated for $20 \mathrm{~h}$ at $37^{\circ} \mathrm{C}$. Samples were prepared with different combinations of the 5 endo- and exoglycosidases. The partly deglycosylated samples were resolved by SDS-PAGE and visualized by Coomassie Brilliant Blue R-250 and PAS staining. Furthermore, the samples were analyzed by Western blotting as described above using polyclonal antibodies against bovine MUC15.

\section{Lectin Blotting}

The structure of the carbohydrate moieties of MUC15 was investigated by a lectin-binding assay using DIGlabeled lectins (Roche Diagnostics) according to the manufacturer's instructions. Briefly, native and partly deglycosylated MUC15 was separated on 18\% SDSpolyacrylamide gels and transferred to Hybond-P PVDF membranes. The membranes were blocked, washed, and incubated with the DIG-labeled lectins. Following a wash, the blots were incubated with alkaline phosphatase-conjugated polyclonal sheep anti-DIG antibodies $(0.75 \mathrm{U} / \mathrm{mL})$. The lectins were visualized with nitro blue tetrazolium chloride/5-bromo-4-chloro3 -indolyl-phosphate. The specificity of the lectin binding was tested with positive control glycoproteins with known oligosaccharide structures: carboxypeptidase Y, transferrin, fetuin, and asialofetuin. Bovine serum albumin was used as a negative control protein. Each lectin recognizes a specific carbohydrate structure: $G a$ - lanthus nivalis agglutinin (GNA) specific to terminal mannose $\alpha(1-3)-, \alpha(1-6)-$, or $\alpha(1-2)-$ linked to mannose; Sambucus nigra agglutinin (SNA) directed to sialic acid $\alpha(2-6)$ linked to galactose or GalNAc; Maackia amurensis agglutinin (MAA), which recognizes sialic acid linked $\alpha(2-3)$ to GlcNAc; peanut agglutinin (PNA) directed to $\mathrm{Gal} \beta(1-3)$ GalNAc without further substituents; and Datura stramonium agglutinin (DSA) specific to galactose $\beta(1-4)$ linked to GlcNAc. Dilutions used were 1:1000 for GNA, SNA, and DSA; 1:200 for MAA; and 1:100 for PNA. Prior to lectin binding, MUC15 samples were treated with PNGase F, neuraminidase, or both using the conditions described for the enzymatic deglycosylation method.

\section{Carbohydrate Composition Analysis of MUC15-Associated Glycans}

The carbohydrate composition analysis of native and PNGase F-treated MUC15 was carried out by combined high-pH anion-exchange chromatography using a CarboPac PA1 column $(4 \times 250 \mathrm{~mm}$; Dionex, Sunnyvale, CA) and pulsed electrochemical detection as described by Pallesen et al. (2001). Because there was linearity between the injected amounts of monosaccharide standards and the peak areas, amounts of the individual monosaccharides in the samples were deduced from standard curves. The exact amount of MUC15 in the sample subjected to monosaccharide composition analysis was determined by AA analysis. The PNGase F (Roche Diagnostics) treatment of MUC15 was performed in $50 \mathrm{~m} M$ sodium phosphate, $\mathrm{pH} 7.5,5 \mathrm{mM}$ dithioerythritol, $2 \%$ octyl-glucopyranoside and incubated for $18 \mathrm{~h}$ at $37^{\circ} \mathrm{C}$. The PNGase F-treated protein was examined by SDS-PAGE and was PAS-stained using standard procedures. Prior to carbohydrate composition analysis of the $\mathrm{N}$-deglycosylated protein, the reaction mixture was thoroughly dialyzed against water to remove octyl-glucopyranoside and the released $\mathrm{N}$ linked glycans.

\section{RESULTS}

\section{Quantification of MUC15 in Milk Fractions}

The amount and distribution of MUC15 in bovine milk and fractions thereof were determined by density scanning of antibody reactivity on Western blots (Table 1). In raw milk, MUC15 constituted $0.08 \%$ of the total protein, and the highest relative content of MUC15 was found in laboratory-made buttermilk (2.23\%). Upon precipitation of MFGM from acidified buttermilk $(\mathrm{pH}=$ 4.8) by centrifugation $(17,000 \times g, 90 \mathrm{~min}), \mathrm{MUC15}$ constituted $1.55 \%$ of the protein in MFGM and as much as $0.88 \%$ of the protein left in the supernatant. A similar 
Table 1. Quantification of MUC15 in different bovine milk samples ${ }^{1}$

\begin{tabular}{lccc}
\hline & $\begin{array}{c}\text { MUC15 } \\
\text { polypeptide, } \\
\mu \mathrm{g} / \mathrm{mg} \text { of } \\
\text { total protein }\end{array}$ & SD & $\begin{array}{c}\text { Weight, } \\
\%\end{array}$ \\
Milk fraction ${ }^{2}$ & 0.80 & 0.09 & 0.08 \\
\hline Raw milk & 0.67 & 0.16 & 0.07 \\
Skim milk & 0.49 & 0.14 & 0.05 \\
Whey & 22.33 & 3.01 & 2.23 \\
Buttermilk & 8.84 & 0.97 & 0.88 \\
Supernatant of acidified buttermilk & 15.45 & 4.67 & 1.55 \\
MFGM (from acidified buttermilk) & 0.92 & 0.30 & 0.09 \\
Ultracentrifuged & 3.93 & 0.50 & 0.39 \\
Serum & - & - & - \\
Skim milk membranes & 9.02 & 1.39 & 0.90 \\
CN & 14.89 & 3.43 & 1.49 \\
Supernatant of buttermilk & 6.86 & 1.02 & 0.69 \\
MFGM (from buttermilk) & - & - & - \\
Dairy industry & - & - & - \\
WFC & 0.16 & 0.04 & 0.02 \\
WPC & 0.82 & 0.16 & 0.08 \\
WPI & & & \\
Buttermilk (from the butter-making machine) & & & \\
Whey (from yellow cheese production) & & & \\
\hline 1'Results are given as average values (n = 4 to 13), micrograms of MUC15 polypeptide/milligrams of total \\
protein and weight percentage. \\
$\quad{ }^{2}$ MFGM = milk fat globule membrane; WFC = whey fat concentrate; WPC = whey protein concentrate; \\
WPI = whey protein isolate.
\end{tabular}

distribution of MUC15 in the MFGM and supernatant fractions, 1.49 and $0.90 \%$, respectively, was observed upon ultracentrifugation of buttermilk. Interestingly, serum and the skim milk membrane fractions obtained by ultracentrifugation of skim milk also contained MUC15, showing their presence outside the traditional lipidous fractions. Among the samples investigated from the dairy industry, whey fat concentrate milk powder showed the highest amount of MUC15 (0.69\%). Notably, industrial buttermilk showed a relatively low content of MUC15 (0.02\%), whereas MUC15 in cheese whey amounted to a level comparable to that of acid whey prepared in the laboratory $(0.08$ and $0.05 \%$, respectively).

\section{O-Sialoglycoprotein Endopeptidase Susceptibility}

O-Sialoglycoprotein endopeptidase from $M$. haemolytica has been shown to specifically cleave mucin-like proteins bearing clusters of O-linked sialoglycans, whereas exclusively $\mathrm{N}$-glycosylated, desialylated or nonglycosylated proteins have not been found to be substrates (Abdullah et al., 1992). All glycoproteins cleaved by $\mathrm{O}$-sialoglycoprotein endopeptidase contain mucinlike regions; however, by no means will all proteins containing O-glycans be cleaved, because nonmucin Oglycosylated proteins, such as fetuin, are not cleaved (Cladman et al., 1996). Thus, we examined whether bovine MUC15 is susceptible to this enzyme and found that O-sialoglycoprotein endopeptidase cleaved bovine
MUC15, leaving 2 bands of approximately 40 and 70 $\mathrm{kDa}$ (Figure 1). Even though this result confirms the presence of clusters of O-linked sialoglycans, information about the nature of these glycans is still limited.

\section{Enzymatic Deglycosylation}

To investigate structural aspects of the O-linked glycans, MUC15 was subjected to enzymatic deglycosylation by sequential addition of each of the 5 enzymes: PNGase F, $\alpha$-2(3,6,8,9)-neuraminidase, O-glycosidase, $\beta(1-4)$-galactosidase, and $\beta$ - $N$-acetylglucosaminidase. A slight decrease in electrophoretic mobility was observed upon incubation with 2(3,6,8,9)-neuraminidase, which catalyzes the hydrolysis of all terminal branched and unbranched sialic acids (Figure 2, lane 2). The mobility was not affected by digestion of native MUC15 with O-glycosidase (Figure 2, lane 3), which hydrolyzes only the Ser- and Thr-linked unsubstituted disaccharide Gal $\beta 1-3$ )GalNAc (Core 1; Endo and Kobata, 1976). However, when O-glycosidase was coincubated with neuraminidase, a clear band shift appeared (Figure 2, lane 4).

Enzymatic removal of the N-linked glycans was achieved with PNGase F, which is a glycan-Asn-amidase specifically cleaving at the innermost GlcNAc of all N-linked oligosaccharides unless they carry $\alpha(1-3)$ linked core fucose residues (Tretter et al., 1991). Removal of $\mathrm{N}$-linked sugars by PNGase $\mathrm{F}$ reduced the molecular weight from $130 \mathrm{kDa}$ to approximately 80 


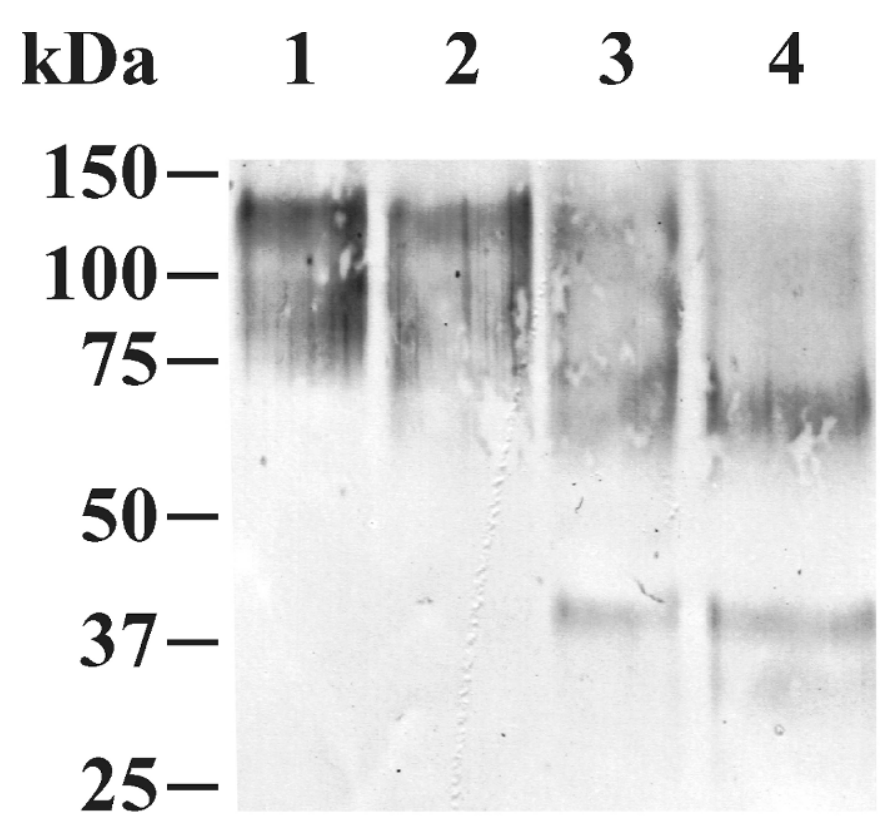

Figure 1. Proteolysis of MUC15 by the O-sialoglycoprotein endopeptidase of Mannheimia haemolytica. Western blot probed with polyclonal antibodies against bovine MUC15. Lane 1, bovine MUC15 control; lane 2, MUC15 treated with O-sialoglycoprotein endopeptidase for $15 \mathrm{~min}$; lane 3, MUC15 treated with O-sialoglycoprotein endopeptidase for $2.5 \mathrm{~h}$; lane 4, MUC15 treated with O-sialoglycoprotein endopeptidase for $21 \mathrm{~h}$. Positions of molecular weight markers are indicated to the left.

$\mathrm{kDa}$ as judged by SDS-PAGE (Figure 3, lane 2). Further supplementation with neuraminidase reduced the electrophoretic mobility because of the removal of sialic acid (Figure 3, lane 3). Treatment with neuraminidase and PNGase F seems to improve the accessibility to cleavage sites for the O-glycosidase, because incubation with all 3 enzymes resulted in a significant reduction of the apparent molecular mass (Figure 3, lane 4). Further deglycosylation of the remaining O-glycans was carried out by addition of either $\beta(1-4)$-galactosidase or $\beta-N$ acetylglucosaminidase to PNGase $\mathrm{F}$, neuraminidase, and O-glycosidase-treated MUC15 (Figure 3, lanes 5 and $6, \quad$ respectively). $\quad \beta-N$-Acetylglucosaminidase cleaves all terminally $\beta$-linked GlcNAc residues and the galactosidase is $\beta(1-4)$ specific, because a nonspecific galactosidase would remove the $\beta(1-3)$ Gal from Core1 structures, leaving O-linked GalNAc, which cannot be removed by O-glycosidase. Apparently, the relative molecular mass did not change after treatment with $\beta(1-4)$-galactosidase or $\beta$ - $N$-acetylglucosaminidase; however, when both enzymes were added simultaneously, a small shift appeared (Figure 3, lane 7).

\section{Lectin Blotting}

The high affinity and narrow specificity of lectins for defined oligosaccharide structures (Cummings, 1994)

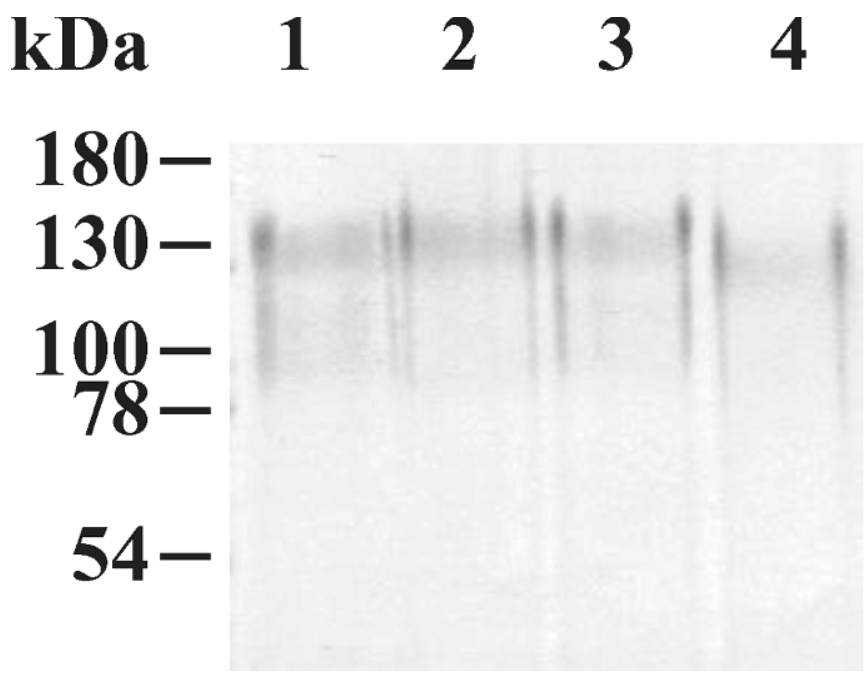

Figure 2. Enzymatic deglycosylation of bovine MUC15. Analysis was performed on $18 \%$ Tris-glycine gels and stained with periodic acid Schiff's reagent. Lane 1, bovine MUC15; lane 2, neuraminidasetreated MUC15; lane 3, O-glycosidase-treated MUC15; lane 4, neuraminidase and O-glycosidase-treated MUC15. Positions of molecular weight markers are indicated to the left.

were used to describe the glycans attached to MUC15. The GNA lectin has high affinity for terminal mannose $\alpha(1-3)-, \alpha(1-6)-$, or $\alpha(1-2)$-linked to mannose, and is thereby suitable for identifying high-mannose type Nlinked glycans (Shibuya et al., 1988). The observed weak interaction between GNA and bovine MUC15 (Figure 4A, lane 1) indicated a minor presence or lack of N-linked high-mannose type glycans. However, preincubation of MUC15 with neuraminidase substantially augmented binding of GNA to MUC15 (Figure 4A, lane 3). Assessing GNA binding following PNGase $\mathrm{F}$ digestion of MUC15 showed no interaction (Figure $4 \mathrm{~A}$, lane 2). This result is consistent with the prior removal of N-linked oligosaccharide chains, because it is generally accepted that O-linked glycans are mannose deficient.

The PNA lectin is specific for the unsubstituted Gal $\beta(1-3)$ GalNAc disaccharide (Lotan et al., 1975), which forms the Core- 1 structure of many O-glycans. Furthermore, Chacko and Appukuttan (2001) have shown that terminal galactose-linked $\alpha(1-3)$ or $\alpha(1-6)$ to penultimate galactose is an efficient ligand for PNA. Peanut agglutinin showed very weak interaction with native and PNGase F-treated MUC15 (Figure 4B, lanes 1 and 2). Upon pretreatment of MUC15 with neuraminidase alone or in combination with PNGase F, a significant increase in PNA interaction with MUC15 was observed (Figure 4B, lanes 3 and 4). The N-linked glycans, however, contributed little to the PNA binding, because the staining intensity appeared to be equal upon re- 
A

$\begin{array}{llllllll}\mathrm{kDa} & 1 & 2 & 3 & 4 & 5 & 6 & 7\end{array}$

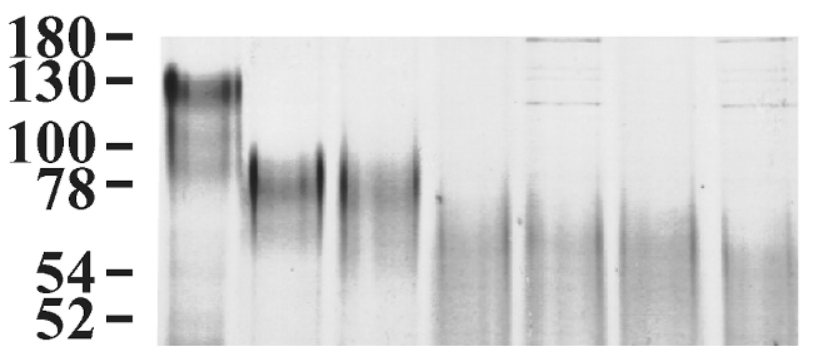

B

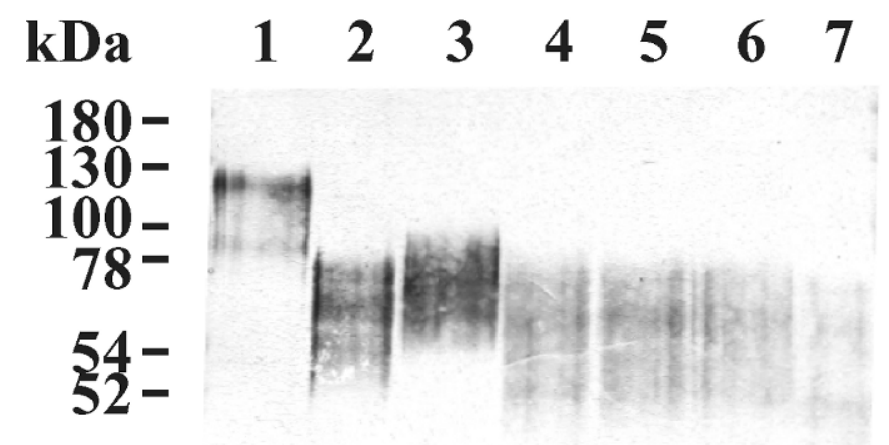

Figure 3. Enzymatic deglycosylation of bovine MUC15. Analysis was performed on $18 \%$ Tris-glycine gels. A) Gel stained with periodic acid Schiff's reagent and Coomassie Brilliant Blue. B) Western blot probed with polyclonal antibodies against bovine MUC15. Lane 1, bovine MUC15; lane 2, peptide:N-glycosidase F (PNGase F)-treated MUC15; lane 3, PNGase F- and neuraminidase-treated MUC15; lane 4, PNGase F-, neuraminidase-, and O-glycosidase-treated MUC15; lane 5, PNGase F-, neuraminidase-, O-glycosidase-, and $\beta(1-4)$-galactosidase-treated MUC15; lane 6, PNGase F-, neuraminidase-, O-glycosidase-, and $\beta$ - $N$-acetylglucosaminidase-treated MUC15; lane 7, bovine MUC15 treated with all 5 enzymes. Positions of molecular weight markers are indicated to the left.

moval of the N-linked glycans. The protein content in the neuraminidase-treated samples was reduced to onetenth to obtain satisfactory PNA blots, implying the presence of a large number of O-glycans with sialylated $\alpha$-linked galactose or simple sialylated Core-1 structures.

Terminal Gal $\beta(1-4)$ GlcNAc ( $N$-acetyllactosamine) is recognized by DSA along with oligomers containing repeating $N$-acetyllactosamine sequences (Crowley et al., 1984). The strong interaction between MUC15 and DSA (Figure 4C, lane 1) showed the presence of $N$-acetyllactosamine units. Upon removal of N-linked glycans with PNGase F, DSA binding activity was drastically reduced (Figure 4C, lane 2). To investigate the presence of terminal sialylated $N$-acetyllactosamine structures, neuraminidase-treated MUC15 was assayed and showed DSA binding activity similar to native MUC15 (Figure 4C, lane 3). Likewise, removal of sialic acid from O-linked glycans (PNGase F treated) did not appear to further increase DSA binding (Figure 4C, lane 4).

The terminal sialic acids were examined using 2 different linkage-specific sialic acid-binding lectins, MAA and SNA. It has been shown that MAA has a structural requirement for the trisaccharide sequence Neu5Ac $\alpha(2-$ 3)Ga1 $\beta(1-4) G 1 c N A c$ (Knibbs et al., 1991), whereas SNA requires the disaccharide structure $\mathrm{Neu} 5 \mathrm{Ac} \alpha(2-6) \mathrm{Gal} /$ GalNAc (Shibuya et al., 1987). MUC15 reacted strongly with SNA, demonstrating the presence of sialic acid terminally linked $\alpha(2-6)$ to galactose or GalNAc (Figure $4 \mathrm{E}$, lane 1), whereas a weak reaction with MAA was detected (Figure 4D, lane 1). Binding of SNA was still observed upon removal of the N-linked glycans; however, the intensity of the staining was greatly reduced (Figure 4E, lane 2). This shows that sialic acid $\alpha(2-6)$ linked to Gal/GalNAc is found in both $\mathrm{N}$ - and O-glycans and that the major part is $\mathrm{N}$-linked. Upon PNGase $\mathrm{F}$ treatment, MAA binding showed no change in intensity compared with native MUC15 (Figure 4D, lane 2). Finally, SNA and MAA binding to neuraminidase-treated samples were performed to determine the degree of desialylation. Consistent with the prior removal of sialic acid, no binding of SNA and MAA was detected (results not shown).

\section{Carbohydrate Composition Analysis of MUC15-Associated Glycans}

The monosaccharides of mildly hydrolyzed native and PNGase F-treated MUC15 were separated and quantified by high-pH anion-exchange chromatography monitored by pulsed electrochemical detection. The carbohydrates linked to bovine milk MUC15 were found to be composed of the 6 monosaccharides fucose, GalNAc, GlcNAc, galactose, mannose, and sialic acid, giving the carbohydrate unit ratio 1:4:6:5:4:5 (Table 2). Marked differences were seen in the ratios of GalNAc and galactose after PNGase $\mathrm{F}$ treatment. In addition, mannose was not detected upon removal of the N-linked glycans, indicating that an exhaustive enzymatic cleavage had been achieved. Thereby, the O-linked glycans contained approximately equal amounts of GalNAc, GlcNAc, galactose, and sialic acid (Table 2).

In total, the carbohydrate was determined to amount to $296 \mathrm{~mol} / \mathrm{mol}$ of MUC15, giving a calculated molecular weight of the carbohydrate of 60.6 and $94 \mathrm{kDa}$ as the total molecular weight of MUC15 (33,317 Da, apomucin). Accordingly, the carbohydrates thereby constituted $65 \%$ of the total molecular weight of MUC15. 
A

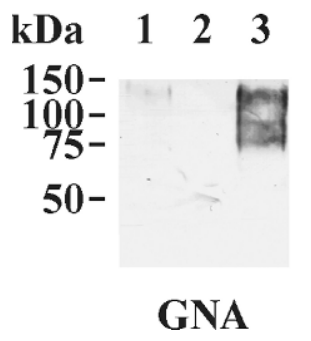

B

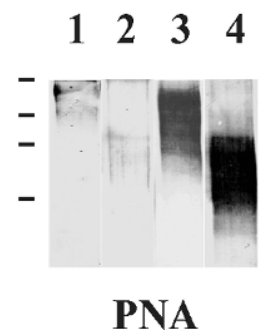

C

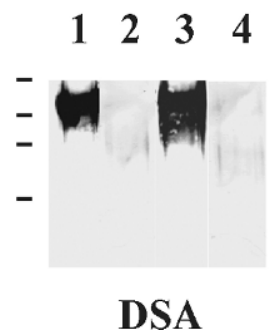

D

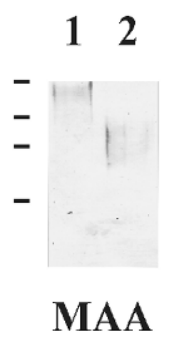

E

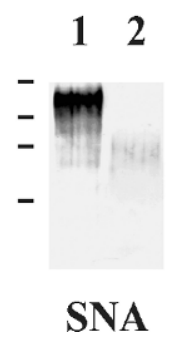

Figure 4. Immunoblot analysis of MUC15 from bovine milk with lectins of the Digoxigenin (DIG) Glycan Differentiation kit (Roche Diagnostics, Mannheim, Germany). Protein samples were separated by SDS-PAGE on 18\% Tris-glycine polyacrylamide gels and blotted onto Hybond-P polyvinylidene difluoride membrane. Blots were sequentially incubated with DIG-labeled lectins, alkaline phosphataseconjugated anti-DIG antibody, and nitro blue tetrazolium chloride/5-bromo-4-chloro-3-indolyl-phosphate as described in the materials and methods section. A) Galanthus nivalis agglutinin (GNA); B) peanut agglutinin (PNA); C) Datura stramonium agglutinin (DSA); D) Maackia amurensis agglutinin (MAA); E) Sambucus nigra agglutinin (SNA). Two micrograms of MUC15 was used in each protein sample except in B, lanes 3 and 4, where $0.2 \mu \mathrm{g}$ was used. Lane 1, MUC15; lane 2, PNGase F-treated MUC15; lane 3, neuraminidase-treated MUC15; lane 4, neuraminidase- and PNGase F-treated MUC15. Positions of molecular weight markers are indicated to the left of each gel.

Normally, using SDS-PAGE the molecular weight of MUC15 is estimated to be approximately $130 \mathrm{kDa}$; however, large variations in the electrophoretic migration are often seen, ranging from approximately 115 to 140 $\mathrm{kDa}$. This phenomenon is frequently observed and most likely reflects the poor SDS-binding capacity of the carbohydrate moieties on massively glycosylated proteins.

\section{DISCUSSION}

In accordance with lipophilic appearance of MUC15, the highest contents of the mucin were found in the fat-containing milk fractions MFGM and buttermilk (1.55 and 2.23\%, respectively). Previously, another MFGM-associated mucin, MUC1, was found to constitute $1.21 \%$ of the proteins in bovine MFGM (Kvistgaard et al., 2004). This is in accordance with the PAS-staining behavior of these 2 mucins, which implies the presence of comparable amounts of MUC1 and MUC15 in bovine MFGM. Upon precipitation of MFGM from buttermilk by centrifugation, MUC15 was still present in

Table 2. Carbohydrate composition analysis of peptide:N-glycosidase F (PNGase F)-treated and native bovine MUC15 ${ }^{1}$

\begin{tabular}{lcc}
\hline & \multicolumn{2}{c}{ Carbohydrate composition, mol \% } \\
\cline { 2 - 3 } & & $\begin{array}{c}\text { PNGase } \\
\text { F-treated } \\
\text { Monosaccharide }\end{array}$ \\
\hline Fucose & MUC15 & 3.80 \\
$N$-Acetylgalactosamine & 3.86 & 24.27 \\
$N$-Acetylglucosamine & 16.73 & 24.79 \\
Galactose & 24.27 & 24.94 \\
Mannose & 19.64 & - \\
Sialic acid & 15.25 & 22.20 \\
\hline
\end{tabular}

${ }^{1}$ Results of the analysis are given as average values $(n=6)$ and mole percentages. the supernatant. This may be explained by the presence of residual MFGM fragments in the supernatant that had not been precipitated. However, application of ultracentrifugation did not markedly change the distribution of MUC15 between the pellet and supernatant. Although MUC15 is an integral membrane protein, the data suggest that a substantial amount of MUC15 in bovine milk was recovered in the aqueous milk phases, such as skim milk, whey, and the supernatant of buttermilk. In fact, skim milk revealed only a slight difference in staining density in favor of whole milk. Similar results have been obtained for bovine MUC1, and it has been suggested that collection, cooling, and mild agitation of milk induce the release of MFGM-associated proteins (Peterson et al., 1998; Kvistgaard et al., 2004). Moreover, the proposed secreted variant MUC15/S (Pallesen et al., 2002) may account for the observation of MUC15 in the supernatants of buttermilk, skim milk, and whey.

Enzymatic deglycosylation experiments and lectin blotting were performed to obtain structural information on the $\mathrm{N}$ - and O-linked sugar moieties of bovine milk MUC15. An overview of the results obtained with lectins is given in Table 3. Interestingly, the successive glycosidase treatments led to an increased smearing appearance of the mucin upon SDS-PAGE. This might have been due to differences in the degree of glycosylation, variations in glycan structures (microheterogeneity), and enzymatic efficiency all resulting in an overall mass heterogeneity.

The presence of mucin-like glycosylations with clusters of sialylated O-linked glycans on bovine MUC15 was documented using O-sialoglycoprotein endopeptidase. Furthermore, treatment of MUC15 with neuraminidase alone or in combination with PNGase F prior 
Table 3. Summary of lectin binding to native and enzymatic deglycosylated bovine milk MUC15 ${ }^{1}$

\begin{tabular}{|c|c|c|c|c|c|}
\hline Lectin & Carbohydrate specificity & MUC15 & $\begin{array}{l}\text { MUC15 + } \\
\text { PNGase F }\end{array}$ & $\begin{array}{l}\text { MUC15 + } \\
\text { Neuramd. }\end{array}$ & $\begin{array}{l}\text { MUC15 + } \\
\text { PNGase F + } \\
\text { Neuramd. }\end{array}$ \\
\hline Galanthus nivalis agglutinin (GNA) & $\operatorname{Man} \alpha(1-3)-, \alpha(1-6)-$, or $\alpha(1-2)-$ linked to Man & + & - & ++ & $\mathrm{NI}$ \\
\hline Datura stramonium agglutinin (DSA) & $\mathrm{Gal} \beta(1-4)$ GlcNAc oligomers & +++ & + & +++ & + \\
\hline Sambucus nigra agglutinin (SNA) & Sialic acid $\alpha(2-6)$ linked to galactose or GalNAc & ++ & + & - & NI \\
\hline Maackia amurensis agglutinin (MAA) & Sialic acid $\alpha(2-3)$ linked to $\mathrm{Gal} \beta(1-4)$ GlcNAc & + & + & - & NI \\
\hline
\end{tabular}

${ }^{1}$ The carbohydrate specificities are outlined by use of simplified structures: mannose (Man); galactose (Gal); $N$-acetylgalactosamine (GalNAc); $N$-acetylglucosamine (GlcNAc); sialic acid; neuraminidase (Neuramd.). PNGase F = peptide:N-glycosidase F; + = degree of positive lectin staining; - = no detectable lectin binding; NI = not investigated.

to O-glycosidase demonstrated the presence of simple sialylated Core-1 O-linked structures. Because the relative mass contribution of the Core- 1 disaccharide (365.3 Da) was detectable by SDS-PAGE, the mono-, di-, or trisialylated Core-1 structures must constitute a large part of the O-linked glycans present on bovine MUC15.

The most commonly occurring modifications of the core Gal $\beta(1-3)$ GalNAc of mucin-type O-glycosylations contain galactose and GlcNAc, as well as terminal sialic acid (Hanisch, 2001). Addition of the exoglycosidases $\beta(1-4)$ galactosidase and $\beta$ - $N$-acetylglucosaminidase to the $\mathrm{N}$ - and partly O-deglycosylated protein should therefore enable cleavage of complex Core- 1 and Core2 O-linked carbohydrates, including those containing poly- $N$-acetyllactosamine units $[\mathrm{Gal} \beta(1-4) \mathrm{GlcNAc}]$, a typical backbone structure of mucins in human milk (Hanisch et al., 1989). Individually they showed no effect, but when both enzymes were added, a small mobility shift appeared, showing that some structures containing $\beta(1-4)$-linked galactose and GlcNAc were present. The slightly reduced molecular weight might have been caused by either improved accessibility for the Oglycosidase or by the simultaneous removal of terminal galactose and GlcNAc.

Treatment of bovine MUC15 with PNGase F demonstrated the ample presence of N-linked glycans. Further knowledge of the N-linked glycans of MUC15 could be extracted from the lectin studies. The GNA lectin, recognizing terminal $\operatorname{Man} \alpha(1-3)$ Man units, showed a huge increase in binding upon removal of sialic acid. These findings are in accordance with the results of others (Shibuya et al., 1988; Hart et al. 2002) and imply the main presence of hybrid-type N-linked oligosaccharides, whereas the number of high-mannose type glycans seemed to be low or absent. Accordingly, those authors suggest that the inability of GNA to bind terminal mannose units in the high-mannose antennas of hybrid-type $\mathrm{N}$-glycans is due to steric hindrance or the impact of the negative charge conferred by terminally linked sialic acids on the complex antennas of hybridtype N-glycans.
From the DSA lectin studies, it is clear that the terminal $N$-acetyllactosamine structures recognized in bovine MUC15 are primarily N-linked, and they appear not to be sialylated. Both Crowley et al. (1984) and Yamashita et al. (1987) have demonstrated that DSA with high affinity binds a branched nonsubstituted pentasaccharide including $2 \mathrm{~N}$-acetyllactosamine disaccharides linked to mannose, Gal $\beta(1-4) \mathrm{GlcNAc} \beta(1-6 /$ 4) $[\mathrm{Gal} \beta(1-4) \mathrm{GlcNAc} \beta(1-2)] \mathrm{Man}$. It was shown that DSA also binds bi-, tri-, and tetraantennary sugar chains in which at least one $\mathrm{Gal} \beta(1-4)$ GlcNAc repeating unit is present in an outer chain. Thus, the present DSA and GNA lectin data suggest that bovine MUC15 contains some hybrid-type $\mathrm{N}$-glycans with terminal sialic acid antennas and some with terminal nonsubstituted $\mathrm{Gal} \beta(1-4) \mathrm{GlcNAc} \beta 1-6 / 4)[\mathrm{Gal} \beta(1-4) \mathrm{GlcNAc} \beta(1-$ $2)]$ Man structures or at least one terminal $\mathrm{Gal} \beta(1-$ 4)GlcNAc repeating unit.

Bovine tissues contain glycoproteins with terminal galactose in both $\alpha$ - and $\beta$-anomeric linkages. Thus, from the weak interaction of PNA with native and Ndeglycosylated MUC15, it must be concluded that MUC15 contains some unsubstituted Gal $\beta(1-3)$ GalNAc structures or terminal Gal $\alpha(1-3 / 6) \mathrm{Gal}$ or both. Neuraminidase treatment of native and PNGase F-treated MUC15 substantially increased binding of PNA, suggesting the abundant presence of sialylated Core-1 Oglycans. This was confirmed by the enzymatic deglycosylation experiments using neuraminidase and O-glycosidase, which demonstrated the presence of a large number of sialylated Core-1 O-glycans as well. Because the monosaccharide composition analysis of the $\mathrm{O}$ linked glycans showed approximately equal amounts of GalNAc, Gal, GlcNAc, and sialic acid, we thereby conclude that, in addition to containing simple sialylated Core- 1 structures, MUC15 contains more complex O-glycans, with GlcNAc containing backbone structures. Core- 1 and Core- 2 based poly- $N$-acetyllactosamine backbones with a high degree of fucosylation represent the predominant glycan species on lactating breast epithelium (Hanisch et al., 1989). Therefore, we specu- 
late that the remaining glycans may contain poly- $N$ acetyllactosamine structures. Lectin blotting with DSA, however, showed that the O-linked glycans contain very few $N$-acetyllactosamine units. It should be noted that peripheral structures other than sialic acid, such as, $\alpha$ fucose, $\alpha$-galactose, $\alpha$-GalNAc, and $\alpha$-GlcNAc (Hanisch, 2001), might block the $N$-acetyllactosamine structures from interaction with DSA (Yamashita et al., 1987).

Bovine MUC15 showed differential reactivity with the 2 linkage-specific sialic acid-binding lectins, SNA and MAA, which recognize $\alpha(2-6)$ and $\alpha(2-3)$ sialylgalactosyl residues, respectively. The lectin SNA showed strong binding to native MUC15 compared with MAA, whereas similar binding was observed to the PNGase F-treated mucin. This indicates a predominance of $\alpha(2-$ 6)-linked sialic acid in the N-linked sialoglycans of MUC15. Knibbs et al. (1991) investigated the ability of the 2 sialic acid-binding lectins to recognize sialylated Core- 1 structures and found that MAA and SNA do not react with the sialylated Core- 1 structures $\operatorname{Neu} 5 \mathrm{Ac} \alpha(2-$ 3) Gal $\beta(1-3)$ GalNAc $\alpha$ and $\operatorname{Gal} \beta(1-3)[\operatorname{Neu} 5 \mathrm{Ac} \alpha(2-$ 6)]GalNAc, respectively. Thus, we were unable to type the sialic acid linkages found on the simple Core-1 structures. However, the SNA lectin-binding assay showed the presence of O-linked terminal Neu5Ac $\alpha(2-$ 6) Gal/GalNAc, and MAA binding demonstrated the presence of $\alpha(2-3)$ sialylated $N$-acetyllactosamine residues within the O-linked glycans.

The monosaccharide composition analysis showed that sialic acid constituted $20 \%$ of the sugar moieties. However, neuraminidase treatment resulted in only a slight decrease in the mobility of MUC15 upon SDSPAGE. Despite the slight mobility change of MUC15, lectin blotting using the sialic acid-binding lectins, SNA and MAA, revealed complete removal of sialic acid (results not shown). This implies that the loss of negative charge caused by desialylation apparently had a greater effect on the mobility of the mucin than the loss of mass. It should be noted that variations in electrophoretic migration of neuraminidase-treated proteins were observed on a case-by-case basis, as described for native MUC15.

The carbohydrate composition of the 2 bovine milk mucins, MUC1 and MUC15, appear to differ. Most notably, MUC15 contains a substantially higher amount of GlcNAc than MUC1, and only the N- and O-linked glycans of MUC15 contain fucose (Pallesen et al., 2001). Thus, the glycosylations of these 2 transmembrane bovine MFGM mucins must be markedly different despite their similarities with regard to localization at the apical plasma membrane, degree of glycosylation, and AA composition of the heavily glycosylated extracellular mucin domain.
The current knowledge about MUC15 is still limited, and the physiological significance of the protein remains elusive; however, ideas may arise from facts about its massive glycosylation. There are 2 principally different roles of extracellular protein-bound glycans. Specific carbohydrate epitopes can serve as ligands for receptors that mediate recognition events, or glycan structures can mediate changes in the biophysical properties of a protein, such as charge, solubility, folding, or susceptibility to proteases (Varki, 1993). As a result of lacking evolutionary pressure to conserve site-specific O-glycosylated Ser and Thr residues, it has been proposed that mucin-type glycosylation in most cases is a bulk property, occurring on Ser and Thr in disordered and surface-exposed regions with little overall sequence conservation (Julenius et al., 2005). In line with that, sequence analysis of MUC15 shows that it holds equivalent structural properties, implying that the function of the dense glycosylation in the mucin domain may primarily be to change the biophysical properties of the mucin. Thus, the mucin glycans provide protective barriers, provide lubrication because of their water-binding capacity, and change the overall structure of the glycosylated region into an extended conformation. It is also an open question whether the mucin-type glycosylation of MUC15, beyond biophysical properties, can modulate various aspects of protein function. Indeed, forthcoming studies are needed before it will be possible to unravel the function of the mucin.

\section{ACKNOWLEDGMENTS}

We express our thanks to Margit Skriver Rasmussen and Anni Boisen for technical assistance and Arla Innovation Centre (Brabrand, Denmark) for supplying the milk samples. This work was supported by the Danish Government (Innovation Law) and the Danish Dairy Research Foundation.

\section{REFERENCES}

Abdullah, K. M., E. A. Udoh, P. E. Shewen, and A. Mellors. 1992. A neutral glycoprotease of Pasteurella haemolytica A1 specifically cleaves O-sialoglycoproteins. Infect. Immun. 60:56-62.

Benfeldt, C., L. B. Larsen, J. T. Rasmussen, P. A. Andreasen, and T. E. Petersen. 1995. Isolation and characterization of plasminogen and plasmin from bovine milk. Int. Dairy J. 5:517-592.

Chacko, B. K., and P. S. Appukuttan. 2001. Peanut (Arachis hypogaea) lectin recognizes $\alpha$-linked galactose, but not $N$-acetyllactosamine in N-linked oligosaccharide terminals. Int. J. Biol. Macromol. 28:365-371.

Christlet, T., and K. Veluraja. 2001. Database analysis of O-glycosylation sites in proteins. Biophys. J. 80:952-960.

Cladman, W. M., M. A. Watt, J. P. Dini, and A. Mellors. 1996. The Pasteurella haemolytica O-sialoglycoprotein endopeptidase is inhibited by zinc ions and does not cleave fetuin. Biochem. Biophys. Res. Commun. 220:141-146. 
Crowley, J. F., I. J. Goldstein, J. Arnarp, and J. Lönngren. 1984. Carbohydrate binding studies on the lectin from Datura stramonium seeds. Arch. Biochem. Biophys. 231:524-533.

Cummings, R. D. 1994. Use of lectins in analysis of glycoconjugates. Methods Enzymol. 230:66-86.

Endo, Y., and A. Kobata. 1976. Partial purification and characterization of an endo-alpha- $N$-acetylgalactosaminidase from the culture of medium of Diplococcus pneumoniae. J. Biochem. (Tokyo) $80: 1-8$.

Gerken, T. A., J. Zhang, J. Levine, and A. Elhammer. 2002. Mucin core O-glycosylation is modulated by neighboring residue glycosylation status. Kinetic modeling of the site-specific glycosylation of the apo-porcine submaxillary mucin tandem repeat by UDPGalNAc:polypeptide $N$-acetylgalactosaminyltransferases T1 and T2. J. Biol. Chem. 277:49850-49862.

Hanisch, F. G. 2001. O-Glycosylation of the mucin type. Biol. Chem. 382:143-149.

Hanisch, F. G., G. Uhlenbruck, J. Peter-Katalinic, H. Egge, J. Dabrowski, and U. Dabrowski. 1989. Structures of neutral O-linked polylactosaminoglycans on human skim milk mucins. A novel type of linearly extended poly- $N$-acetyllactosamine backbones with Gal beta(1-4)GlcNAc beta(1-6) repeating units. J. Biol. Chem. 264:872-883.

Hart, M. L., M. Saifuddin, K. Uemura, E. G. Bremer, B. Hooker, T. Kawasaki, and G. T. Spear. 2002. High mannose glycans and sialic acid on gp120 regulate binding of mannose-binding lectin (MBL) to HIV type 1. AIDS Res. Hum. Retroviruses 18:13111317.

Heegaard, C. W., L. B. Larsen, L. K. Rasmussen, K.-E. Hojberg, T. E. Petersen, and P. A. Andreasen. 1997. Plasminogen activation system in human milk. J. Pediatr. Gastroenterol. Nutr. 25:159-166.

Ho, J. J., R. S. Jaituni, S. C. Crawley, S. C. Yang, J. R. Gum, and Y. S. Kim. 2003. N-Glycosylation is required for the surface localization of MUC17 mucin. Int. J. Oncol. 23:585-592.

Hvarregaard, J., M. H. Andersen, L. Berglund, J. T. Rasmussen, and T. E. Petersen. 1996. Characterization of glycoprotein PAS-6/7 from membranes of bovine milk fat globules. Eur. J. Biochem. 240:628-636.

Julenius, K., A. Mølgaard, R. Gupta, and S. Brunak. 2005. Prediction, conservation analysis and structural characterization of mammalian mucin-type O-glycosylation sites. Glycobiology 15:153-164.

Knibbs, R. N., I. J. Goldstein, R. M. Ratcliffe, and N. Shibuya. 1991. Characterization of the carbohydrate binding specificity of the leukoagglutinating lectin from Maackia amurensis. J. Biol. Chem. 266:83-88.

Kvistgaard, A. S., L. T. Pallesen, C. F. Arias, S. Lopez, T. E. Petersen, C. W. Heegaard, and J. T. Rasmussen. 2004. Inhibitory effects of human and bovine milk constituents on rotavirus infections. J. Dairy Sci. 87:4088-4096.

Lotan, R., E. Skutelsky, D. Danon, and N. Sharon. 1975. The purification, composition, and specificity of the anti-T lectin from peanut (Arachis hypogaea). J. Biol. Chem. 250:8518-8523.

Mather, I. H., C. B. Tamplin, and M. G. Irving. 1980. Separation of the proteins of bovine milk-fat-globule membrane by electrofocusing with retention of enzymatic and immunological activity. Eur. J. Biochem. 110:327-336.

Moniaux, N., F. Escande, N. Porchet, J. P. Aubert, and S. K. Batra. 2001. Structural organization and classification of the human mucin genes. Front. Biosci. 6:d1192-d1206.

Pallesen, L. T., M. H. Andersen, R. L. Nielsen, L. Berglund, T. E. Petersen, L. K. Rasmussen, and J. T. Rasmussen. 2001. Purification of MUC1 from bovine milk-fat globules and characterization of a corresponding full-length cDNA clone. J. Dairy Sci. 84:2591-2598.

Pallesen, L. T., L. Berglund, L. K. Rasmussen, T. E. Petersen, and J. T. Rasmussen. 2002. Isolation and characterization of MUC15, a novel cell membrane-associated mucin. Eur. J. Biochem. 269:2755-2763.

Peterson, J. A., S. Patton, and M. Hamosh. 1998. Glycoproteins of the human milk fat globule in the protection of the breast-fed infant against infections. Biol. Neonate 74:143-162.

Rose, M. C., and J. A. Voynow. 2006. Respiratory tract mucin genes and mucin glycoproteins in health and disease. Physiol. Rev. 86:245-278.

Schacterle, G. R., and R. L. Pollack. 1973. A simplified method for the quantitative assay of small amounts of protein in biological material. Anal. Biochem. 51:654-655.

Shibuya, N., I. J. Goldstein, W. F. Broekaert, M. Nsimba-Lubaki, B. Peeters, and W. J. Peumans. 1987. The elderberry (Sambucus nigra L.) bark lectin recognizes the $\operatorname{Neu} 5 \operatorname{Ac}(\alpha 2-6) \mathrm{Gal} / \mathrm{GalNAc}$ sequence. J. Biol. Chem. 262:1596-1601.

Shibuya, L. T., I. J. Goldstein, E. J. M. Van Damme, and W. J. Peumans. 1988. Binding properties of a mannose-specific lectin from the snowdrop (Galanthus nivalis) bulb. J. Biol. Chem. 263:728-734.

Tretter, V., F. Altmann, and L. Marz. 1991. Peptide- $N 4-(N$-acetylbeta-glucosaminyl)asparagine amidase $\mathrm{F}$ cannot release glycans with fucose attached alpha 1-3 to the asparagine-linked $N$-acetylglucosamine residue. Eur. J. Biochem. 199:647-652.

Varki, A. 1993. Biological roles of oligosaccharides: All of the theories are correct. Glycobiologi 3:97-130.

Yamashita, K., K. Totani, T. Ohkura, S. Takasaki, I. J. Goldstein, and A. Kobata. 1987. Carbohydrate binding properties of complextype oligosaccharides on immobilized Datura stramonium lectin. J. Biol. Chem. 262:1602-1607. 